\title{
Review Article \\ Follow-Up in Head and Neck Cancer: A Management Dilemma
}

\author{
Francesca De Felice, ${ }^{1}$ Daniela Musio, ${ }^{1}$ and Vincenzo Tombolini ${ }^{1,2}$ \\ ${ }^{1}$ Department of Radiotherapy, Policlinico Umberto I, “Sapienza” University of Rome, Viale Regina Elena 326, 00161 Rome, Italy \\ ${ }^{2}$ Spencer-Lorillard Foundation, Viale Regina Elena 262, Rome, Italy \\ Correspondence should be addressed to Francesca De Felice; fradefelice@hotmail.it
}

Received 18 October 2014; Revised 14 December 2014; Accepted 28 December 2014

Academic Editor: Tino Just

Copyright (C) 2015 Francesca De Felice et al. This is an open access article distributed under the Creative Commons Attribution License, which permits unrestricted use, distribution, and reproduction in any medium, provided the original work is properly cited.

Follow-up program in squamous cell carcinoma of head and neck district is crucial to detect locoregional recurrence and second primary tumors and to manage treatment toxicities. The choice of the appropriate frequency of visits and imaging modality can be troublesome. Details of timing surveillance and type of diagnostic procedure are still not well defined. This review highlights the problem from a clinician's point of view.

\section{Introduction}

A review of recent developments of follow-up management for head and neck $(\mathrm{H} \& \mathrm{~N})$ cancer is presented. Considering the multiple site and histological classification of H\&N cancer, we focus on nasopharynx and squamous cell carcinoma, which includes primary sites in oral cavity, hypopharynx, oropharynx, and larynx. Although the prognosis of H\&N cancer depends on primary tumor site, the posttreatment evaluation is common in all $\mathrm{H} \& \mathrm{~N}$ district.

The design of follow-up program is a relatively recent research area. In the past few years the concept of conservative treatments became the primary end-point. The appropriate management has been driven not only by innovations in the delivery of medical care, but also by developments in technology tools $[1,2]$. On the other hand, posttreatment follow-up gains importance in clinical performance. Optimal form of surveillance is not clear and data regarding costeffectiveness of rigorous surveillance are lacking [3]. Despite the timing protocol and the modalities used varying considerably among clinicians, the aim of a prompt detection and treatment of recurrent disease, as well as second primaries, is a common denominator. Nowadays, follow-up should be performed by a multiprofessional team, with expertise in management and prevention of treatment toxicities. Current modalities for follow-up include complete $H \& N$ clinical exam and structural examinations.

\section{Evidence of Follow-Up}

Treatment failure in $\mathrm{H} \& \mathrm{~N}$ cancer after surgery and/or (chemo)radiotherapy is primarily associated with locoregional recurrence disease. Therefore posttreatment surveillance should be focused on detecting tumor recurrence at an early stage in order to provide a salvage procedure $[4,5]$.

2.1. Recurrent Tumor. Cancer recurrence is defined as the reemerge of disease after treatment and after a 6-month period of complete regression [6]. In $\mathrm{H} \& \mathrm{~N}$ cancer, local recurrence data vary from $<10 \%$ to about $50 \%$, based on primary site and stage [7-10]. Surveillance programs are based on the conventional assumption that early recurrence detection is related to an increased survival. Actually, benefit in cancer survival with routine surveillance has not been well proven in literature. Most studies concluded that regular follow-up does not influence survival outcomes [8, 9, 11-13]. With careful follow-up, recurrence could be detected, but there is no evidence to support a real improvement in survival, in the physician-detected recurrent disease versus patient-detected [13, 14]. Only one retrospective analysis [9] resulted in better survival rates in detection of recurrence with physical examination in comparison with the selfreferral recurrence (58 months versus 32 months, resp.; $P$ value $<0.05)$. This analysis is discordant with other studies reported in literature. Flynn et al. [14] have shown that 
TABLE 1: Guidelines for follow-up intervals for head and neck cancers.

\begin{tabular}{|c|c|c|c|c|c|c|}
\hline & \multicolumn{2}{|c|}{$\mathrm{NCCN}$} & \multicolumn{2}{|c|}{ AIRO } & \multicolumn{2}{|c|}{ BAHNO } \\
\hline & Clinical exam & Imaging & Clinical exam & Imaging & Clinical exam & Imaging \\
\hline Year 1 & Every 1-3 mo & Within $6 \mathrm{mo}$ & Every 1-2 mo & Every 6 mo & Every $4-6$ we & Within $3 \mathrm{mo}$ \\
\hline Year 2 & Every 2-6 mo & - & Every 2-3 mo & Every 6 mo & Every 4-6 we & - \\
\hline Year 3 & Every 4-8 mo & - & Every 4-6 mo & $-^{*}$ & Every 3 mo & - \\
\hline Year 4 & Every 4-8 mo & - & Every 4-6 mo & $-^{*}$ & Every $6 \mathrm{mo}$ & - \\
\hline Year 5 & Every $4-8 \mathrm{mo}$ & - & Every $4-6$ mo & - & Every 6 mo & - \\
\hline$>5$ years & Every 12 mo & - & Every 6-12 mo & - & Every $12 \mathrm{mo}$ & - \\
\hline
\end{tabular}

* If nasopharynx, RM every 6 months for 4 years.

NCCN: National Comprehensive Cancer Network; AIRO: Associazione Italiana Radioterapia Oncologica; BAHNO: British Association of Head and Neck Oncologists; mo: month; we: week.

an adequate follow-up frequency is important to evaluate treatment results, to manage late complication, and to offer emotional support. They conducted a retrospective analysis of 223 patients with advanced $\mathrm{H} \& \mathrm{~N}$ cancer treated with curative intent. In early physician detection of locoregional recurrence, no improvement in survival was demonstrated.

Because most recurrences are reported by the patient, a training to recognise symptoms or signs should be paramount. Increased local pain, development of a new lump in the neck, unexplained weight loss, and increased difficulty in swallowing suggest recurrence. Most recurrences appear within 18 months, but late recurrences may appear after 5 years, especially for laryngeal primaries [7].

2.2. Second Primary Tumour. Patients with previous $\mathrm{H} \& \mathrm{~N}$ malignancy run a high risk (10-20\%) of developing second primary cancer in lung or upper aerodigestive tract, due to the same risk factors, chronic nicotine and alcohol intake. Although this evidence is well documented in literature, the impact of early detection on survival is not clear, considering their poor prognosis [15-17].

\section{Head and Neck Cancer Surveillance Protocols}

Follow-up of patients with H\&N cancer, firstly, should focus on early detection of locoregional recurrence and management of adverse symptoms or posttreatment complications. Appropriate follow-up program should be performed according to guidelines. At present several guidelines are available, but there is insufficient evidence to recommend one guideline to others. Table 1 summarizes the main schedules of followup in literature.

3.1. National Comprehensive Cancer Network. The National Comprehensive Cancer Network (NCCN) guidelines recommend complete clinical exam, including fiberoptic examination, every 1 to 3 months for the first year, every 2 to 6 months for the second year, every 4 to 8 months for the third to fifth years, and annually thereafter. A posttreatment baseline imaging exam within 6 months of RT/CRT treatment is recommended for T3-4 or N2-3 cancer of the oropharynx, hypopharynx, glottic and supraglottic larynx, and nasopharynx only. The NCCN guidelines believe that it is safe and reasonable to perform further imaging investigation based on symptoms or signs. Patients with smoking history should be monitored by chest imaging [18].

3.2. European Society for Medical Oncology. Recommendation for $\mathrm{H} \& \mathrm{~N}$ cancers' surveillance is also provided by European Society for Medical Oncology (ESMO) Guidelines Working Group. Clinical and imaging exams should be included to evaluate treatment response, but timing is not specified. It is possible to deduce that imaging exams should be performed in case of clinical suspicion of recurrence. A posttreatment baseline imaging exam of primary localization and neck is recommended [19].

3.3. Associazione Italiana Radioterapia Oncologica. Practice guidelines issued by Associazione Italiana Radioterapia Oncologica (AIRO) group recommended endoscopic exam every 1-2 months for year 1, every 2-3 months for year 2, every 4-6 months for years 3-5, and then annually. Based on primary tumor localization, MRI or CT is indicated every 6 months for 2 years (for 4 years in nasopharynx) and then if there are symptoms. In the case of an uncertain diagnosis, a PET/TC exam [20] is recommended.

3.4. British Association of Head and Neck Oncologists. The British Association of Head and Neck Oncologists (BAHNO) scheduled follow-up visits every 4-6 weeks during the first and the second year after therapy, every 3 months for year 3 , every 6 months for years 4-5, and then annually. Imaging baseline study is recommended 3 months after completion of treatment [21].

\section{General Clinical and Imaging Evaluation}

Posttreatment evaluation, both clinical and radiologic, is necessary to detect tissue modifications. During followup examinations the major difficulty is distinguishing local recurrence from treatment toxicity, as a result of surgery or radiation-induced tissue distortions. 
4.1. Clinical Exam. Local control clinical assessment is an inexpensive method to follow up patients after treatment. It is paramount after completing treatment, even if it should be difficult due to treatment effects on mucosa. Because of anatomic changes, such as fibrosis and lymph edema, the efficacy of clinical examination, especially in palpation, could make detection of recurrence difficult. On the other hand, these changes, as well as those secondary to flap reconstruction and vascularized scar, could determine high false-positive rates at imaging [22]. Hypothetically a mucosal recurrence may be missed on follow-up routine imagingbased information. Therefore a scrupulous complete H\&N clinical examination, with mirror examination and fiberoptic nasopharyngoscopy, must be performed.

4.2. Imaging Evaluation. Cross-sectional imaging exams are fundamental to evaluate $\mathrm{H} \& \mathrm{~N}$ district. Computed tomography (CT) scan, magnetic resonance (MR) imaging, and 18F-fluorodeoxyglucose positron emission tomography (18-FFDG-PET) are nowadays considered standard for estimating treatment response in $\mathrm{H} \& \mathrm{~N}$ cancer. Which is the modality to choose depends on clinical practice. Generally, the posttreatment baseline imaging exam should be the same that has been used in the pretreatment evaluation [23].

Historically, CT is used to assess posttreatment response in $\mathrm{H} \& \mathrm{~N}$ cancer. Obviously a CT scan performed without contrast is not optimal for $\mathrm{H} \& \mathrm{~N}$ district, because tumor and lymphoid tissue, as well as muscle, have the same density [24].

MR has the advantage over CT to provide a better soft-tissue differentiation. It is recommended in case of nasopharyngeal, base of tongue, sinonasal, or skull base carcinomas [25]. Due to the limited accuracy of both modalities, especially in lymph node evaluation, new functional imaging technologies are being employed. Diffusion weighted imaging (DWI) is an MR technique that has been suggested as noninvasive imaging biomarker to predict tumor response. The obvious advantage of DWI-MR, which uses water molecules motion in intracellular and extracellular space to reflect biological changes in tumour microenvironment, is that it is useful for accurately distinguishing recurrent disease from radiation-induced soft-tissue changes $[5,26]$. DWI-MR should detect the disease before the patient notices a return of any symptom.

The diagnostic accuracy of 18-F-FDG-PET after surgery or (chemo)radiotherapy has been tested in recurrence detention of $\mathrm{H} \& \mathrm{~N}$ cancer. Independently of treatment modality, literature data indicated a better accuracy with 18-F-FDGPET than with CT and/or MRI $(P=0.03)$ [27]. However, the role of 18-F-FDG-PET has been especially investigated in the postradiotherapy surveillance and thus its known accuracy limitations due to treatment induced inflammation [28]. Following definitive (chemo)radiotherapy, a residual CT abnormality in the neck, but a negative PET scan, defined as the absence of metabolic activity, is associated with no nodal progression [29]. FDG-PET should also be helpful in the distinction of tissue necrosis from recurrent tumor [30]. Considering that 18-F-FDG uptake may be observed in normal tissues, such as lymphoid tissue, vocal cord, and cervical muscles, a potential role of other PET radiotracers has been tested. 18-F-Fluorothymidine for DNA synthesis (18-F-FLT) seems to be the most promising due to its high specificity; however its real utility is currently not firmly demonstrated [31]. Therefore PET with the tracer 18-F-FDG is still the standard. To reduce false-positive results, 18-FFDG-PET should be performed at 10-12 weeks after the end of treatment; this time point is also considered an optimal balance between clinical response evaluation and surgical management, if necessary [29].

\section{Point of View}

There are important differences among the guidelines regarding frequency of routine visits and evidence-based data on timing surveillance are lacking. The decision of posttreatment surveillance seems a matter of geography. A randomized trial comparing a specific follow-up strategy with a self-patient referral policy is not yet available.

Many physicians continue to order imaging studies in asymptomatic patients based on believing that earlier detection of relapse will favourably influence the final outcome. The potential benefits of a shorter surveillance than recommended intervals need to be weighed against the potential exposition of patients to unnecessary risks and cost. Certainly routine follow-up is the better chance of managing treatment toxicities and providing reassurance.

\section{Conclusion}

We can conclude that unanimous consensus on optimum posttreatment follow-up in patients with $\mathrm{H} \& \mathrm{~N}$ cancer does not exist. The aim is to adopt a rational follow-up program that reassures patients, minimizes patients discomfort, saves time, and reduces costs. All patients should have routinely nasopharyngoscopy and posttreatment baseline imaging. Imaging with CT or MRI or PET at regular intervals had no advantage over improvement of patients' outcomes.

Prospective studies are needed. The goal would be to avoid being overly aggressive in clear situation while ensuring sufficiently determination in dubious condition.

\section{Conflict of Interests}

The authors declare that there is no conflict of interests regarding the publication of this paper.

\section{References}

[1] J.-P. Pignon, A. L. Maître, E. Maillard, and J. Bourhis, "Metaanalysis of chemotherapy in head and neck cancer (MACHNC): an update on 93 randomised trials and 17,346 patients," Radiotherapy and Oncology, vol. 92, no. 1, pp. 4-14, 2009.

[2] J. L. Lefebvre, "Surgery for laryngopharyngeal SCC in the era of organ preservation," Clinical and Experimental Otorhinolaryngology, vol. 2, no. 4, pp. 159-163, 2009.

[3] R. Kazi, K. Manikanthan, K. A. Pathak, and R. C. Dwivedi, "Head and neck squamous cell cancers: need for an organised time-bound surveillance plan," European Archives of Oto-RhinoLaryngology, vol. 267, no. 12, pp. 1969-1971, 2010. 
[4] S. S. Yom, M. Machtay, M. A. Biel et al., "Survival impact of planned restaging and early surgical salvage following definitive chemoradiation for locally advanced squamous cell carcinomas of the oropharynx and hypopharynx," The American Journal of Clinical Oncology, vol. 28, no. 4, pp. 385-392, 2005.

[5] V. Vandecaveye, F. De Keyzer, S. Nuyts et al., "Detection of head and neck squamous cell carcinoma with diffusion weighted MRI after (chemo)radiotherapy: correlation between radiologic and histopathologic findings," International Journal of Radiation Oncology Biology Physics, vol. 67, no. 4, pp. 960-971, 2007.

[6] B. H. Lok, J. Setton, F. Ho, N. Riaz, S. S. Rao, and N. Y. Lee, "Nasopharynx," in Perez \& Brady's Principles and Practice of Radiation Oncology, E. C. Halperin, L. W. Brady, C. A. Perez, and D. E. Wazer, Eds., chapter 41, pp. 730-759, Lippincott Williams \& Wilkins, 6th edition, 2013.

[7] S. E. Lester and R. G. Wight, "'When will i see you again?' using local recurrence data to develop a regimen for routine surveillance in post-treatment head and neck cancer patients," Clinical Otolaryngology, vol. 34, no. 6, pp. 546-551, 2009.

[8] T. R. Cooney and M. G. Poulsen, "Is routine follow-up useful after combined-modality therapy for advanced head and neck cancer?" Archives of Otolaryngology-Head and Neck Surgery, vol. 125, no. 4, pp. 379-382, 1999.

[9] A. V. M. de Visscher and J. J. Manni, "Routine long-term followup in patients treated with curative intent for squamous cell carcinoma of the larynx, pharynx, and oral cavity: does it make sense?" Archives of Otolaryngology-Head and Neck Surgery, vol. 120, no. 9, pp. 934-939, 1994.

[10] M. A. W. Merkx, J. J. M. Van Gulick, H. A. M. Marres et al., "Effectiveness of routine follow-up of patients treated for T1$2 \mathrm{~N} 0$ oral squamous cell carcinomas of the floor of mouth and tongue," Head and Neck, vol. 28, no. 1, pp. 1-7, 2006.

[11] S. Saussez, C. Dekeyser, M. P. Thill, and G. Chantrain, "Importance of clinical and radiological follow-up in head and neck cancers," B-ENT, vol. 3, no. 4, pp. 179-184, 2007.

[12] J. J. Grau, A. Cuchi, J. Traserra et al., "Follow-up study in head and neck cancer: cure rate according to tumor location and stage," Oncology, vol. 54, no. 1, pp. 38-42, 1997.

[13] S. C. Ritoe, P. F. M. Krabbe, J. H. A. M. Kaanders, F. J. A. van den Hoogen, A. L. M. Verbeek, and H. A. M. Marres, "Value of routine follow-up for patients cured of laryngeal carcinoma," Cancer, vol. 101, no. 6, pp. 1382-1389, 2004.

[14] C. J. Flynn, N. Khaouam, S. Gardner et al., "The value of periodic follow-up in the detection of recurrences after radical treatment in locally advanced head and neck cancer," Clinical Oncology, vol. 22, no. 10, pp. 868-873, 2010.

[15] J. A. S. Atienza and C. A. Dasanu, "Incidence of second primary malignancies in patients with treated head and neck cancer: a comprehensive review of literature," Current Medical Research and Opinion, vol. 28, no. 12, pp. 1899-1909, 2012.

[16] S. C. Ritoe, P. F. M. Krabbe, M. M. G. Jansen et al., "Screening for second primary lung cancer after treatment of laryngeal cancer," Laryngoscope, vol. 112, no. 11, pp. 2002-2008, 2002.

[17] N. Nandy and C. A. Dasanu, "Incidence of second primary malignancies in patients with esophageal cancer: a comprehensive review," Current Medical Research and Opinion, vol. 29, no. 9, pp. 1055-1065, 2013.

[18] National Comprehensive Cancer Network, Guidelines Head and Neck Version 2, 2014, http://www.nccn.org/.

[19] V. Grégoire, J.-L. Lefebvre, L. Licitra, and E. Felip, "Squamous cell carcinoma of the head and neck: EHNS-ESMO-ESTRO clinical practice guidelines for diagnosis, treatment and followup," Annals of Oncology, vol. 21, supplement 5, pp. v184-v186, 2010.

[20] "AIOCC-AIRO-AIOM: Tumori della testa e collo: Algoritmi Diagnostico-Terapeutici AIOCC-AIRO-AIOM-Versione 2," 2012, http://www.radioterapiaitalia.it/.

[21] British Association of Head and Neck Oncologists, "Practice care guidance for clinicians participating in the management of head and neck cancer patients in the UK. Drawn up by a Consensus Group of Practising Clinicians," European Journal of Surgical Oncology, vol. 27, supplement A, pp. S1-S17, 2001.

[22] B. P. Sullivan, K. A. Parks, N. R. Dean, E. L. Rosenthal, W. R. Carroll, and J. S. Magnuson, "Utility of CT surveillance for primary site recurrence of squamous cell carcinoma of the head and neck," Head and Neck, vol. 33, no. 11, pp. 1547-1550, 2011.

[23] M. F. Schlumpf and S. Haerle, "The current role of imaging in head and neck cancer: a clinician's perspective," Swiss Medical Weekly, vol. 144, Article ID w14015, 2014.

[24] S. L. Liauw, A. A. Mancuso, R. J. Amdur et al., "Postradiotherapy neck dissection for lymph node-positive head and neck cancer: the use of computed tomography to manage the neck," Journal of Clinical Oncology, vol. 24, no. 9, pp. 1421-1427, 2006.

[25] R. Hermans, "Posttreatment imaging in head and neck cancer," European Journal of Radiology, vol. 66, no. 3, pp. 501-511, 2008.

[26] D. Musio, F. De Felice, A. L. Magnante et al., "Diffusionweighted magnetic resonance application in response prediction before, during, and after neoadjuvant radiochemotherapy in primary rectal cancer carcinoma," BioMed Research International, vol. 2013, Article ID 740195, 5 pages, 2013.

[27] Y. Anzai, W. R. Carroll, D. J. Quint et al., "Recurrence of head and neck cancer after surgery or irradiation: prospective comparison of 2-deoxy-2-[F-18]fluoro-D-glucose PET and MR imaging diagnoses," Radiology, vol. 200, no. 1, pp. 135-141, 1996.

[28] H. Schöder, M. Fury, N. Lee, and D. Kraus, "PET monitoring of therapy response in head and neck squamous cell carcinoma," Journal of Nuclear Medicine, vol. 50, supplement 1, pp. 74S-88S, 2009.

[29] S. V. Porceddu, D. I. Pryor, E. Burmeister et al., "Results of a prospective study of positron emission tomography-directed management of residual nodal abnormalities in node-positive head and neck cancer after definitive radiotherapy with or without systemic therapy," Head and Neck, vol. 33, no. 12, pp. 1675-1682, 2011.

[30] W. F. McGuirt, "Laryngeal radionecrosis versus recurrent cancer," Otolaryngologic Clinics of North America, vol. 30, no. 2, pp. 243-250, 1997.

[31] V. Grégoire and A. Chiti, "Molecular imaging in radiotherapy planning for head and neck tumors," Journal of Nuclear Medicine, vol. 52, no. 3, pp. 331-334, 2011. 


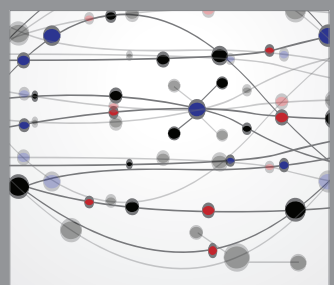

The Scientific World Journal
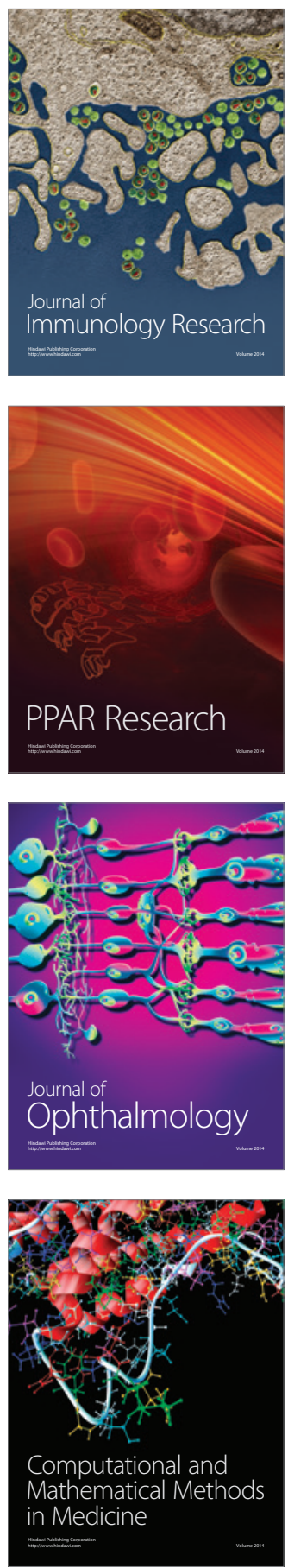

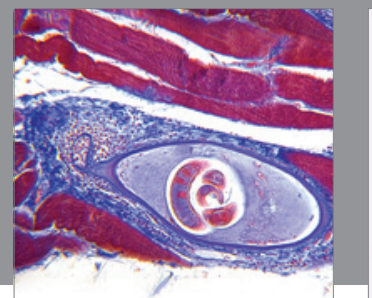

Gastroenterology

Research and Practice
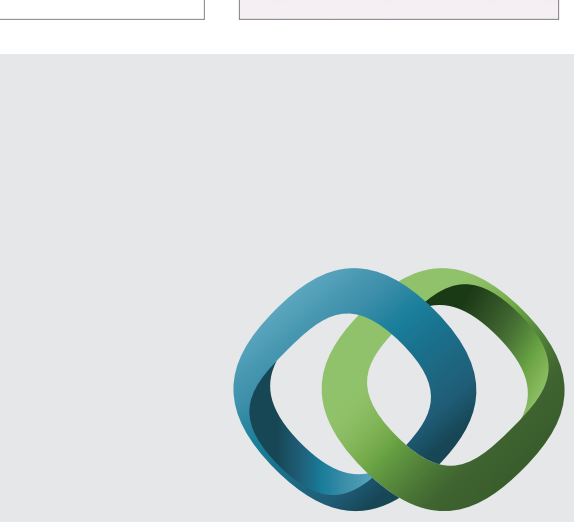

\section{Hindawi}

Submit your manuscripts at

http://www.hindawi.com
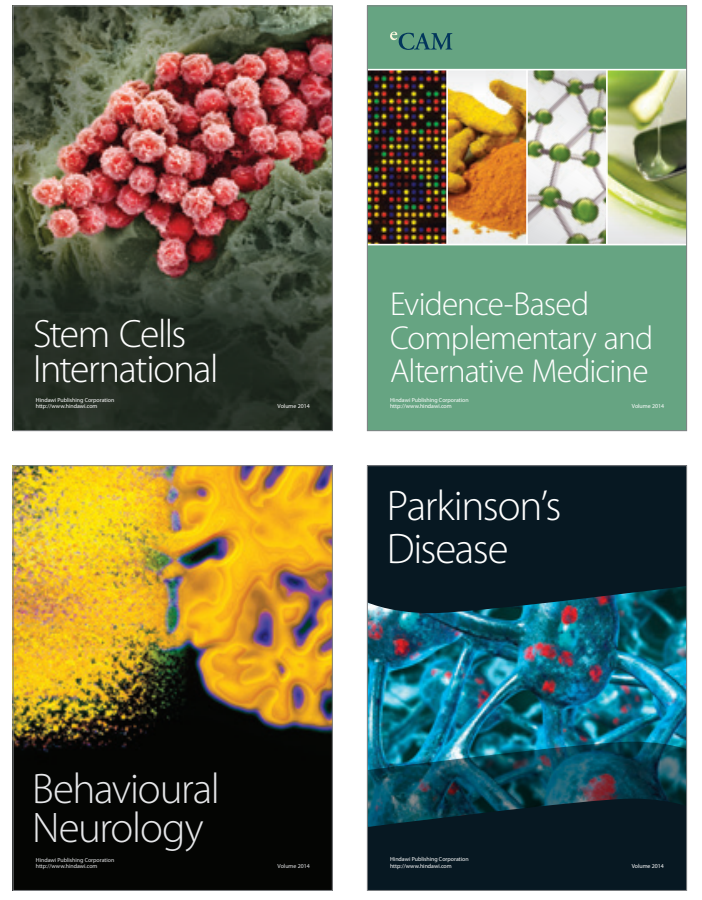
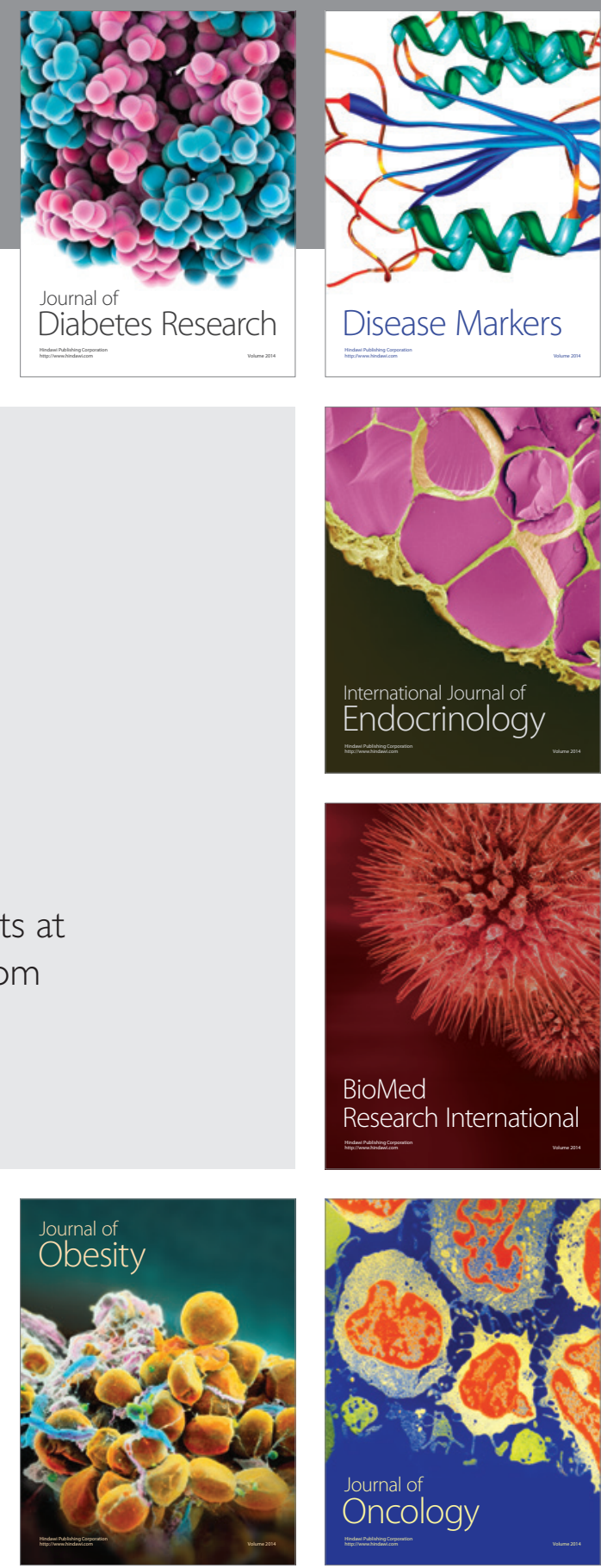

Disease Markers
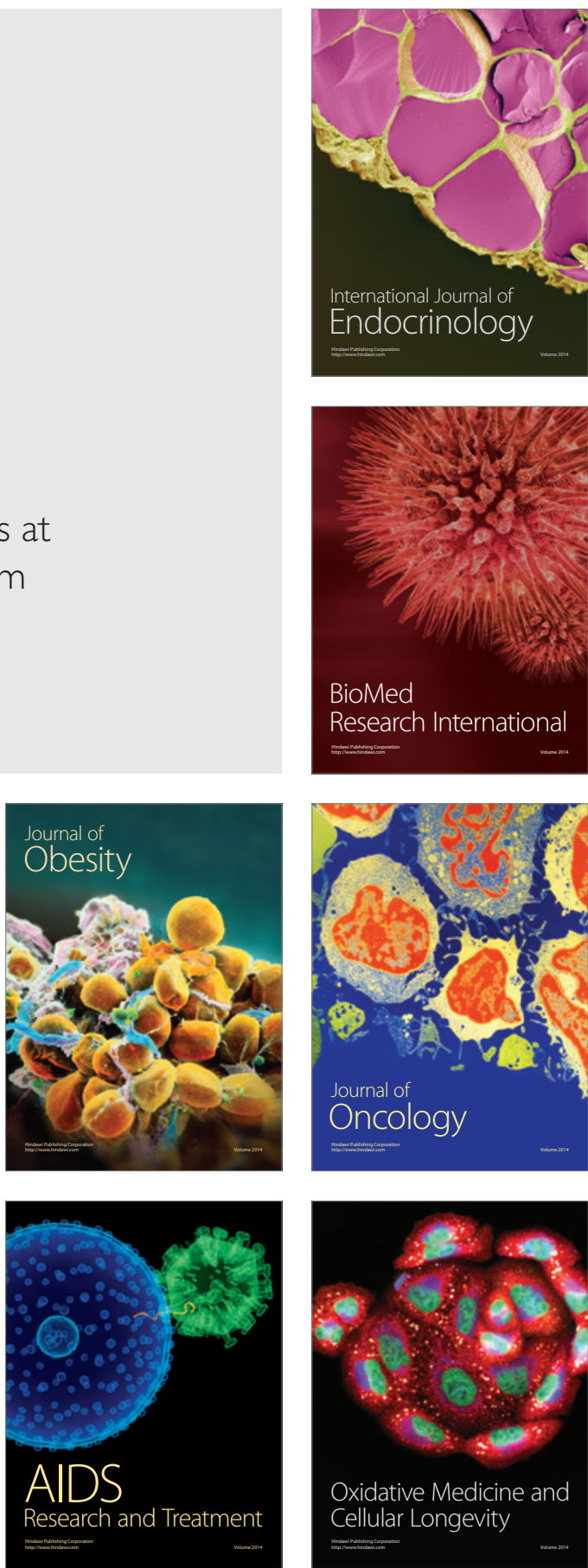